\title{
An Improved Method for Quantifying Liquid Saturation Using Transparent Soil
}

\author{
Juan Wang $\mathbb{D}$, ${ }^{1,2}$ Wei Liu $\mathbb{D}^{1,2}$, Wenwu Chen $\mathbb{D}^{1,2}$ Xuebin Ran, ${ }^{3}$ Yanfang Zhang, ${ }^{1,2}$ \\ and Gaochao Lin iD ${ }^{4}$ \\ ${ }^{1}$ Department of Geological Engineering, Lanzhou University, Tianshui Road, 222, Lanzhou 730000, China \\ ${ }^{2}$ Key Laboratory of Mechanics on Disaster and Environment in Western China, Ministry of Education, China \\ ${ }^{3}$ School of Information Science and Technology, Lanzhou University, Lanzhou 730000, China \\ ${ }^{4}$ School of Civil and Environmental Engineering, University of New South Wales, Sydney, Australia
}

Correspondence should be addressed to Wei Liu; liuw2013@lzu.edu.cn

Received 15 May 2019; Revised 18 July 2019; Accepted 26 August 2019; Published 10 October 2019

Academic Editor: Kirk Hatfield

Copyright ( $\odot 2019$ Juan Wang et al. This is an open access article distributed under the Creative Commons Attribution License, which permits unrestricted use, distribution, and reproduction in any medium, provided the original work is properly cited.

Transparent porous media combined with digital image processing technology can be used to quantify the saturation of local fluids. In this regard, the calibration relationship between the pixel intensity of the image and the saturation of the wetting liquid is the crucial premise for whether this technique can be promoted and applied, and it is also a bottleneck that limits whether this technique can be rapidly and extensively developed. However, owing to gravity-driven effects, wetting liquid in a residual state usually exhibits an irregular boundary caused by the phenomenon of saturation overshoot, which makes it very difficult to use the previous overflow method to obtain the data pair of saturation-pixel intensity accurately. To this end, we have made some improvements to the specific testing method for wetting liquid saturation in the residual state and proposed a local centrifugal sampling method, which considerably improved the accuracy of this method. The results show that the relationship between the pixel intensity and saturation of the transparent soil obtained through this method is generally consistent with the regularity of the previous calibration method but shows better correlation. The results of this study will drive further potential application of transparent soil in the flow of partially saturated porous media.

\section{Introduction}

Soil water content is a key variable in understanding the hydraulic behavior and hydrological processes of unsaturated soils in seepage areas [1-3]. However, as natural soils are opaque under visible light, it is often infeasible to observe percolation processes occurring inside the soil directly, and the use of some specialized instruments with light particles having specific penetrating properties (i.e., $\mathrm{X}$-ray and gamma-ray) or some noninvasive optical methods (e.g., the LTM optical transmission method and the LRM optical perspective method) is often necessary for implementation. Among these methods, the former often has the limitations of expensive construction and complex operation and carries the harm from high-energy radiation, whereas the latter is limited by research area and sample size [4-6]; Oostrom and [7, 8].
Transparent soil consisting of refractive index- (RI-) matched solid particles and pore fluids has become a powerful tool for investigating many geotechnical and global environmental engineering problems [9-12]. In a transparent soil model, the combination of transparent soil and optical imaging techniques allows for noninvasive studies of flow characteristics within the soil continuum. Owing to its good transparency, transparent soil technology has shown unique research value in studies of seepage visualization, and it has progressed rapidly in recent years. So far, some studies have reported the visualization of multiphase fluid interactions in mesoscale seepage. For example, Welker et al. [13] investigated flow properties around prefabricated vertical drains (PVDs). Liu et al. [14] studied flow visualization in two-dimensional tank tests. Lo et al. [15] proposed a new type of water-based transparent material known as aquabeads and used them to simulate the flow in natural soils and 
establish an optical tracking system for fluid motion in a two-dimensional soil profile physical model. Sui et al. [16] and Gao et al. [17] simulated the propagation characteristics of grouting in rock fissures. Tabe [18] studied the migration of non-aqueous phase liquids (NAPL). Theodoropoulou et al. [19] proposed a high-resolution visualization method for the hydrodynamic diffusion of contaminants in transparent glass-etched pore networks. Other researchers have used laser scanning $[20,21]$ or multiple cameras [22] to reconstruct the three-dimensional behavior of seepage. These studies were implemented using PIV technology by adding fluorescent reflective particles to the fluid to produce patterns of image subset correlation. However, the visualization of flow within soil pores is still at an early stage of development, and most studies are limited to certain macroscopic qualitative measurements, such as the width and height of nonwetting liquid intrusion or the time to breakthrough.

The application of transparent soil technology to the determination of fluid saturation is rarely implemented, which is mainly limited to empirical calibration of the relationship between image pixel intensity and saturation. In the above study, saturated transparent soil was used to achieve transparency in the entire process, and the presence of air bubbles is unacceptable. However, this is the advantage of using transparent soil in the phenomenon of unsaturated flow. Transparent soil appears white when it is completely dry, and it is transparent when fully saturated. Between these states, some researchers have worked on the correlation between the pixel intensity and saturation of partially saturated transparent soil [23-26]. The correlation between fluid saturation and digital image pixel intensity values facilitates the potential application of transparent soil in unsaturated soil mechanics. In fact, using the visualized kinematics method, transparent soil technology can effectively yield the water field profile in the unsaturated seepage zone, facilitating dynamic research on the flow characteristics of soil models, which is a significant advance in laboratory permeability testing.

However, owing to some basic properties of indoor seepage testing, some practical difficulties exist in the calibration method of saturation-pixel intensity relationship. For example, in the sample equipment process, it is difficult to determine the initial saturation of $100 \%$ control conditions using the wet pluviation method [23, 27]. Notably, studies have shown that, under certain boundary conditions and initial conditions, a typical "saturation overshoot" of gravity-driven finger flow occurs during indoor column permeability testing [28-30]. This is the result of an unstable drive where the density of the pore fluid is greater than the density of air. The resulting unsaturated seepage profile exhibits nonuniform characteristics (see an example in Figure 1), and combined with the limitations of transient system-related variables, the flow methods used in previous studies often cannot accurately and effectively determine saturation values under residual wetting conditions. This considerably increases the difficulty of determining the calibration relationship between the two, leading to the current slow progress in this area. Therefore, it is of great practical significance to enhance studies of calibration methods between fluid saturation and the corresponding pixel intensity in unsaturated transparent soil.

Therefore, we have improved the calibration method of saturation-pixel intensity relationship to some extent. For saturation, we extracted a partial sample from the uniform humidity portion of the image and then quantified its saturation via centrifugation. For pixel intensity acquisition, we developed a metering program that automatically performs image information processing. Accordingly, a two-dimensional moisture profile of different drainage stages of the soil column was obtained.

\section{Materials and Experimental Technique}

\subsection{Materials}

2.1.1. Transparent Soil. The transparent soil used in this study is a fused quartz sand $[23,25,31]$, comprised of uniformed fine sand with a particle size ranging from $0.5 \mathrm{~mm}$ to $1.0 \mathrm{~mm}$ and a refractive index of 1.459 . The particle size distribution curve obtained by laser particle size analyzer (Malven, MS-2000) is shown in Figure 2. Relevant soil properties are summarized in Table 1 . The specific gravity is 2.19 , and the minimum dry density is $1150 \mathrm{~kg} / \mathrm{cm}^{3}$. The placed densities from multiple tests range from 1200 to $1280 \mathrm{~kg} / \mathrm{cm}^{3}$ and tend to be slightly higher after cycles of filling and drainage, $1295 \mathrm{~kg} / \mathrm{cm}^{3}$, with a corresponding void ratio of 0.70 . The void ratio of transparent soil was obtained according to the following formula:

$$
e=\frac{\rho_{\mathrm{s}}}{\rho_{\mathrm{d}}}-1
$$

where $\rho_{\mathrm{s}}$ is the grain density of transparent sand, $\rho_{\mathrm{s}}=$ $2.19 \mathrm{~g} / \mathrm{cm}^{3}$, and $\rho_{\mathrm{d}}$ is the dry density of transparent sand. According to the data records of soil placement method (dry tremie), we can obtain that $\rho_{\mathrm{d}}=1.293 \mathrm{~g} / \mathrm{cm}^{3}$, so $e=0.70$.

2.1.2. Fluid. The pore fluid in transparent porous media is a mixture of one mineral oil with a refractive index of 1.464 at $20^{\circ} \mathrm{C}$ and $n$-dodecane $\left(\mathrm{C}_{12}\right)$ with a refractive index of 1.422 at $20^{\circ} \mathrm{C}$ (approximately $10: 1, \mathrm{~g} / \mathrm{g}$ ), which equals to that of the fused quartz, 1.459. An Abbe digital handheld refractometer (2WAJ) was used to verify the refractive index of the fluid mixture. The oil mixture had a density of $825 \mathrm{~kg} / \mathrm{m}^{3}$ and an dynamic viscosity of $20.464 \mathrm{cP}$ at $25^{\circ} \mathrm{C}$, measured using a Stabinger viscometer apparatus (Anton Paar, SVM 3000). The contact angle was measured form digital images taken of a droplet of the oil mixture on a level Perspex surface using the pendant drop method on a drop shape analyzer (Kruss, DSA-100). The properties of porous liquids are listed in Table 2.

2.2. Sample Preparative Techniques. Sample preparation greatly affects the quality of transparent soil samples; therefore, the techniques used will produce unique changes 


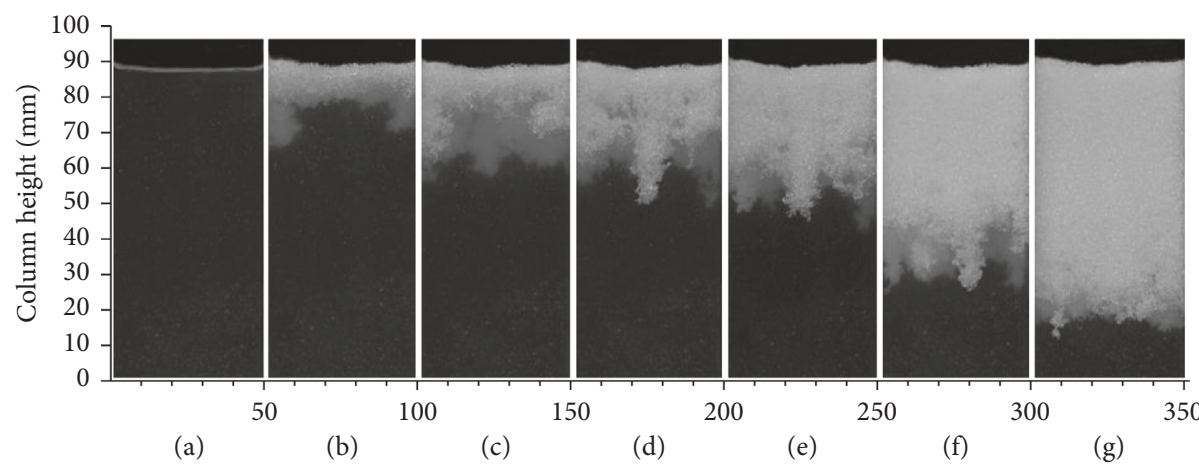

Figure 1: Visualization of the fingering phenomena at the air front through partially saturated transparent soil $(9 \sim 10 \mathrm{~mm}$ saturated transparent soil was filled into a transparent Perspex column (with the internal cross-sectional area of $50.0 \times 50.0 \mathrm{~mm}^{2}$ ) by wet pluviation method, and water profiles were observed at different times during the drainage process: (a) $t=0: 0: 0$, (b) $t=0: 33: 40$, (c) $t=0: 38: 25$, (d) $t=0$ : 42:20, (e) $t=0: 51: 20$, (f) $t=1: 26: 10$, and (g) $t=2: 53: 50$.

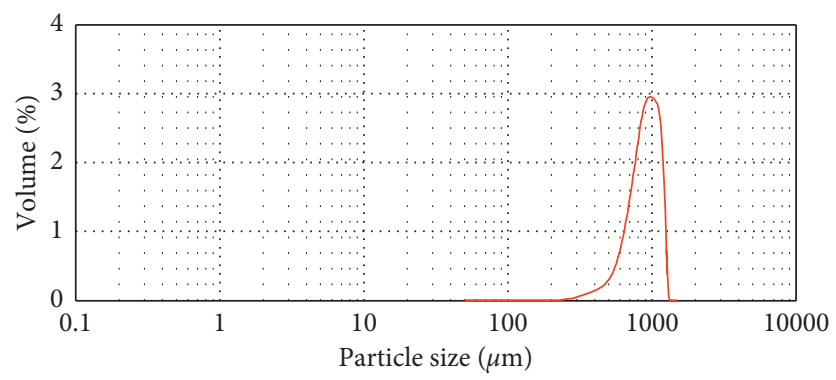

Figure 2: Particle size distribution curve.

TABle 1: Transparent soil properties.

\begin{tabular}{|c|c|c|c|c|}
\hline Parameter & Units & Coarse gradation ${ }^{\mathrm{a}, \mathrm{b}}$ & Fine gradation $^{\mathrm{a}, \mathrm{b}}$ & Finer gradation \\
\hline Refractive index & & 1.459 & 1.459 & 1.459 \\
\hline \multicolumn{5}{|l|}{ Particle size distribution } \\
\hline D10 & $\mathrm{mm}$ & 0.75 & 0.13 & 0.61 \\
\hline D30 & $\mathrm{mm}$ & 1.16 & 0.27 & 0.77 \\
\hline D60 & $\mathrm{mm}$ & 1.75 & 0.45 & 0.94 \\
\hline Coefficient of uniformity & & 2.3 & 3.3 & 1.54 \\
\hline Coefficient of curvature & & 1.0 & 1.2 & 1.03 \\
\hline Minimum dry density & $\mathrm{kg} \cdot \mathrm{m}^{-3}$ & 1050 & - & 1150 \\
\hline
\end{tabular}

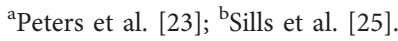

TABLE 2: Oil and water properties.

\begin{tabular}{|c|c|c|c|c|c|c|}
\hline Parameter & Units & Water $^{\mathrm{a}}$ & Mixture oils ${ }^{\mathrm{b}}$ & Mixture oils & Mineral oils & $\mathrm{C}_{12}$ \\
\hline Denstiy & $\mathrm{g} \cdot \mathrm{cm}^{-3}$ & 1.0 & 0.845 & 0.825 & 0.834 & 0.745 \\
\hline Refractive index & - & 1.333 & 1.459 & 1.459 & 1.464 & 1.422 \\
\hline Dynamic viscosity $\left(25^{\circ} \mathrm{C}\right)$ & $\mathrm{cP}$ & 0.89 & 10.1 & 20.464 & 32.507 & 1.280 \\
\hline Kinematic viscosity $\left(25^{\circ} \mathrm{C}\right)$ & $\mathrm{mm}^{2} / \mathrm{s}$ & - & - & 24.791 & 38.987 & 1.719 \\
\hline Contact angle on Perspex & 。 & 53 & 10 & 35.41 & 41.41 & 21.0 \\
\hline
\end{tabular}

${ }^{\mathrm{a}}$ Munson et al. [41]; ${ }^{\mathrm{b}}$ Peters et al. [23].

in soil structure, density, and initial saturation. For this experiment, two sample preparative techniques are listed.

The first technique, wet pluviation, involves initially filling the column with only pore fluid and subsequently allowing the soil particles to fall through the fluid. This installation technique is particularly useful for soil samples at $100 \%$ saturation and minimum density. This method has more consistent results and is less affected by operator influence [27]. However, soil layer constructed in this way may be sorted to a certain degree.

However, the standard method of pluviation can introduce entrapped air into transparent soil, thereby reducing the quality of transparency. Several factors improve the quality of transparent soil samples prepared by means of 
pluviation, such as decreasing the pluviation height and controlling the pluviation rate [32]. These factors primarily reduce the volume of entrapped air in the transparent soil sample.

The second method, dry tremie, where the initial dry soil is placed into the column through a hose running from the top of the column. It is suitable for soil samples with an initially dry moisture content and a close to uniform soil structure, and a loose initial density. In this method, one strategy to prevent bubbles from forming is to introduce porous liquid slowly from the bottom of the sample until the fused quartz is submerged after dry funnel loading [33]. Note the hose should be kept 1 to $4 \mathrm{~cm}$ above the advancing surface of transparent soil to prevent the separation of particles during the placing process.

However, the initial saturation conditions of the test cannot be strictly controlled as neither of these two placing methods can guarantee $100 \%$ saturation of the sample.

\subsection{Experimental Apparatus. Column testing was carried} out using a hanging column instrumented apparatus, as shown in Figure 3. The column was constructed with $18 \mathrm{~mm}$-thick Perspex sheets joined at right angles, with an internal cross-sectional area of $50.0 \times 50.0 \mathrm{~mm}^{2}$ and height of $300 \mathrm{~mm}$.

A Canon Eos digital SLR camera (70D) was used to capture high-resolution photographs of the column. The camera was set one meter in front of the column. Some prime parameters of the camera setting were as follows: a manual " $M$ " shutter speed of $1 / 25 \mathrm{~s}$, an aperture of $\mathrm{F} 1 / 4.5$ and ISO setting of 400 , auto white balance (AWB), and no flash. Lighting is provided by overhead fluorescent lamps. Multiple feature points on both sides of the column were used as reference dots for coordinated purpose. The uneven light was a gradual change, so the exposure operation was also adjusted in a gradually-changing way.

For better image acquisition, the whole experimental setting was surrounded with a black curtain. To reduce the influence of the light source in the environment, black and white reference dots were placed along the column length on both sides, at intervals of $50 \mathrm{~mm}$. The function of reference dots is to align the coordinate system for each image as a continuous color control over the length of the column [23]. Each reference dot consists of a bigger white circle and a black spot in the center [34], whose diameters are $10.0 \mathrm{~mm}$ and $5.0 \mathrm{~mm}$, respectively.

\section{Test Methods}

To relate an image intensity value to the saturation of transparent soil, a more adaptable image calibration procedure was proposed to construct the relationship between direct measurements of saturation using centrifugal sampling and indirect measurements of image pixel intensity carried out by the photometric procedure and fit the parameters. The flow chart of calibration procedure is shown in Figure 4.
This section mainly describes how to determine the saturation of the residual transparent sand in the column using the centrifugal method. Firstly, for local residual saturation of transparent soil, samples were selected within the region of the continuous and homogeneous area in a seepage profile for local sampling. Subsequently, centrifugation was carried out to obtain the oil-sand mass ratio $w$ of the samples to determine the degree of saturation. This operation relieves the otherwise strict condition that the initial water content must be at $100 \%$ saturation when employing the conventional overflow method and overcomes the difficulty that the residual moisture content cannot be quantified due to the nonuniformity of boundary conditions in the seepage process.

Our sampling method is as following. Firstly, determine the approximate position of target region from the scale on the side of the column according to the picture, which can be easily obtained from the transparency of the soil sample. Secondly, dig out the soil sample from the nontarget area from the top, and carefully take the sample from the target area with two small spoons from both directions and then place it into a vessel for later use. Then, the sands containing oil were sampled from the target area and put into the vessel, notated as $m_{1}, g$. Actually, due to the transient effects of the fluid, it is hard to take samples without oil loss in digging. In order to avoid oil loss in digging soil sample out, the digging tools need to be greased in advance, and the whole operation process needs to be skillful and quick enough, which were adequate for the residual state of the transparent sand.

Subsequently, the sand was transferred to a centrifugal bottle, and pure water was added into it, too. And then separate them by high-speed centrifugation. The centrifuge speed was set at $3500 \mathrm{rpm}$, and the degreasing operation was completed after two $30 \mathrm{~min}$ cycles. After centrifugal treatment, the oil and water were separated due to different densities, and the oil phase in the upper layer is removed with a syringe and wiped out carefully. The upper pure water was then further filtered, and the remaining wet sand (at this moment, ideally supposed that only transparent sand and pure water are left) was placed in the oven within a container and dried to a constant weight (the drying temperature set at $105-110^{\circ} \mathrm{C}$, for over $8 \mathrm{~h}$ ). The alcohol combustion method was used to check whether the degreasing was completed.

After that, the weight of the dry sand could be obtained. The oil-sand ratio $w(\mathrm{~g} / \mathrm{g})$ of the selected samples is determined according to the mass before and after centrifugal operation; that is,

$$
w=\frac{m_{1}-m_{\mathrm{d}}}{m_{\mathrm{d}}},
$$

where $m_{1}$ is the weight of local sampling oil sands $(\mathrm{g})$ and $m_{\mathrm{d}}$ is the weight of the dry sand $(\mathrm{g})$. So, the saturation $S_{\mathrm{w}}$ could be calculated according to the following equation:

$$
S_{\mathrm{w}}=\frac{w \cdot G_{\mathrm{s}}}{e \cdot \rho_{\mathrm{l}}},
$$

where $S_{\mathrm{w}}$ is the saturation of the sample (\%); $w$ is the oil-sand ratio $(\mathrm{g} / \mathrm{g}) ; G_{\mathrm{s}}$ is the specific gravity of transparent sand, 


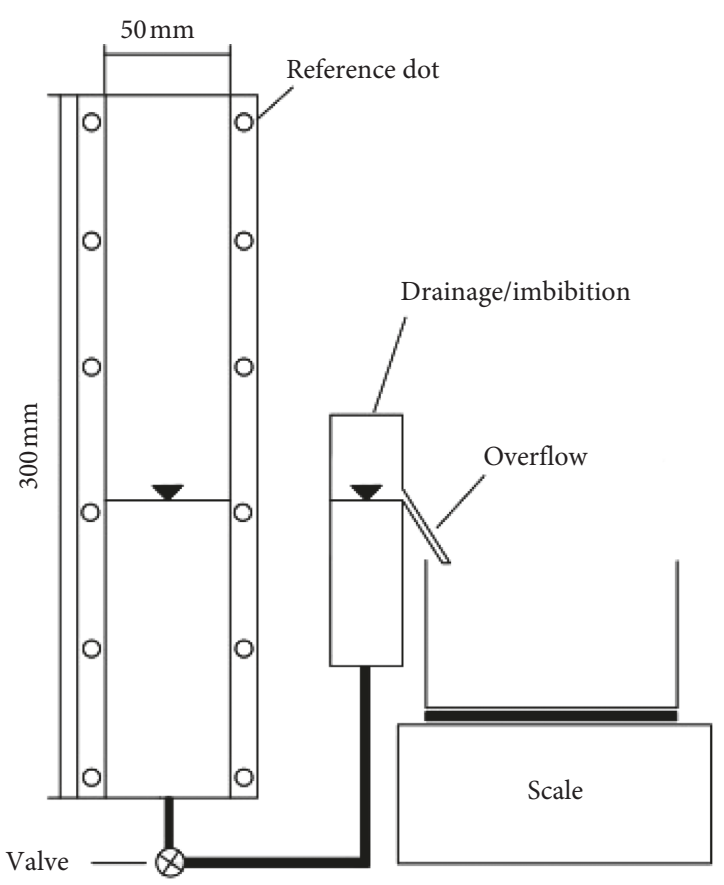

FIGURE 3: Column apparatus for calibration test.

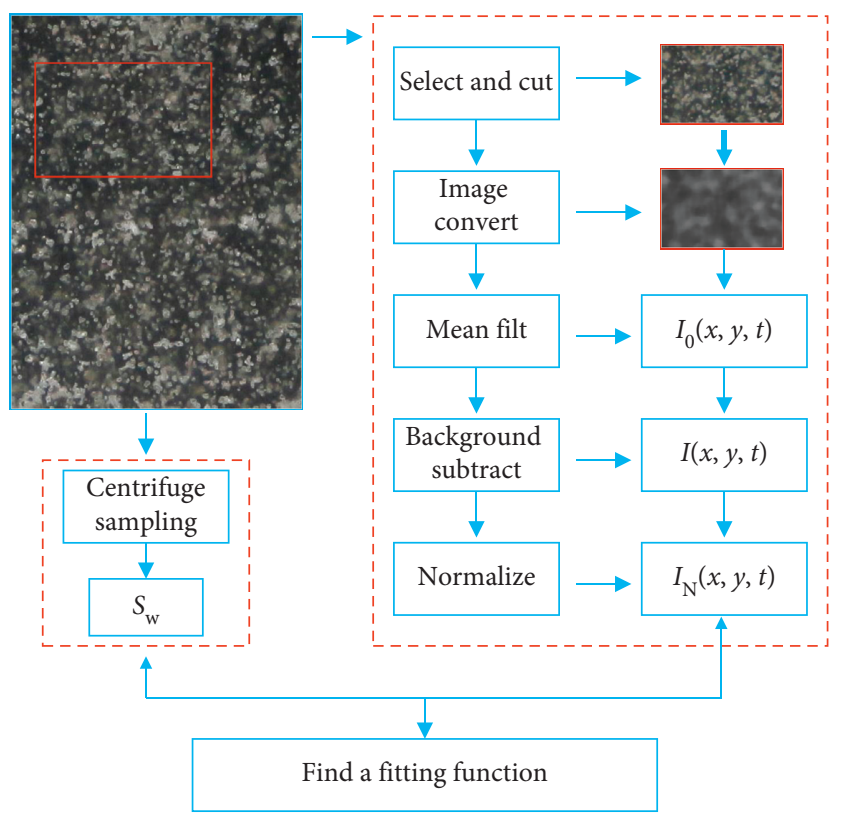

FIgURE 4: The procedure of calibration method.

$G_{\mathrm{s}}=2.19 ; e$ is void ratio of transparent sand, $e=0.7$; and $\rho_{1}$ is the density of mixture oil, $\rho_{1}=0.825$.

It is noted that the the void ratio $e$ is a calculation index, which was obtained according to equation (1) before digging disturbance. Although the sample digging method employed here to measure the saturation will have some influence on the void ratio, this influence on the saturation is less or even negligible, so the saturation still corresponds to the original structural state of the transparent sand in the column. Here, it was assumed that the saturation of samples almost remains the same before and after sample digging. In summary, our target void ratio $e$ only corresponds to the state of the soil sample in the column before digging disturbed. Therefore, the disturbance of sample digging has no influence on the calculation of the void ratio.

In essence, this method is used to measure local water content, similar to a moisture sensor, but more efficient as it obtains the average water content in an entire area, not just for a single point, which is more suitable for this test condition. Moreover, a moisture sensor is usually required to be installed or fixed at a specific location of the soil column in advance, but this location is usually not the test target area. The experimental results showed that the saturation determined by this method was consistent with the results obtained by the traditional overflow method. However, it can dramatically reduce the strict experimental conditions that the overflow method requires for initial saturation.

There are two fluids in the centrifuged sand, one is the original pore fluid-the mixture oil and pure water. Note that the mixture oils are nonwetting fluid, whereas pure water is wetting fluid, which was added in the centrifuge method to achieve oil-water separation effect.

The soil samples in this area were photographed. The pixel intensity was obtained from the pictures of the uniform humidity section. Here, uniform humidity section refers to the uniform area avoiding such nonuniform fingering boundary. The relationship between the pixel intensity and saturation of transparent soil is more reliable.

The corresponding image analysis techniques will be presented in detail in the next section. The resolution of the initial pictures is $5184 \times 3456$. After being converted into gray-scale images, it will make no changes to the resolution. In other words, only the color information will be removed. When it comes to measuring sample saturation in local areas, the picture needs to be clipped. However, no matter what the size of the clipping is, the sharpness of the local photo will not be changed, that is, the quality of the local picture will not be changed. Since the image clipping involved herein is based on the sampling area of local humidity, the size can be very flexible, either big or small. It is because what we need is the average gray-scale value in the clipped area, which uniquely corresponds to the saturation value of the uniform humidity region. Therefore, the gray level identification method is not better with greater resolution values, but highly depends on the better consistency with the actual humidity sampling.

\section{Image Analysis Technique}

An indispensable component of geotechnical modeling with transparent soils involves acquisition and analysis of images. Digital image techniques have been proven to be a very effective noninvasive measurement method in geotechnical engineering research and practice. For example, particle image velocimetry (PIV) $[34,35]$ and digital image correlation (DIC) [36] have been widely used in measuring soil deformation. 
The chart of the image processing method is shown in Figure 4 . Here, we will introduce the image analysis method in detail.

4.1. Basis of Image Analysis. In all optical measurement techniques, image processing is required to convert the reflected or transmitted light intensities into the variable of interest (i.e., saturation, concentration, etc.). The transparent soil samples appear white in the dry state and completely transparent in the fully saturated state. Therefore, when a black background is laid, it turns black in the image when saturated. That is, when transparent soil's saturation varies from $100 \%$ to $0 \%$, a color spectrum from black to white is generated. Therefore, there is a clear basis for the measurement technique for volumetric moisture content based on the image intensity value. The visibility of transparent soil is closely related to the saturation magnitude, which could be explained by Fresnel's law [4]. The greater the saturation of transparent sand, the better the transparent effect. The test results near the saturation part are more reliable compared with those near the dry part. The visible depth is very restricted for observing the nonlinear phenomenon through dry sand. So here, it was assumed that the overall humidity/ saturation is consistent across the depth. As a result, we had to estimate the saturation magnitude behind the dry sand according to the surface saturation.

The situation in reality is far more complex. The pixel intensity of the soil column is a function of the uniformity of light not just moisture content, as reflected light is used as the light source. Therefore, a few corrections must be made to ensure color consistency along the column height. These barriers in pixel intensity can be solved by placing reference dots on both sides of the column to provide color information because the color of the reference dots does not change depending on lighting either from the light source or from the temporary shadows cast on the soil column. Therefore, the pixel intensity of the reference dots can be used to correct changes in pixel intensity that result due to factors other than saturation [22, 23, 37].

4.2. Image Processing Procedure. A photometric program has been developed to automatically process the images according to successive steps involved in cutting, naming, manual exposure, format conversion, denoising, background subtraction, and normalization.

4.2.1. The Batch Program. After the target region in selected images is cut, named, manually exposed, and saved, a batch program will run including format conversion and denoising processes as follows.

Firstly, import the image and extract the information stored in it, and then run the program code to convert each image format into a gray matrix of $0-255$. Secondly, a denoising operation was carried out to remove or reduce the impact of a large number of white particles or noise in the pictures (the pixel is replaced by the mean value of the gray pixel within a certain area, and all pixels are traversed this way). Then, the program will calculate the average gray value of each pixel intensity in the image matrix, with the results automatically stored in the output file. In this way, the average pixel intensity located at position $(x, y)$ on the picture taken at time $t$ within the target measurement area is obtained and denoted as $I_{0}(x, y, t)$.

Mean filtering is a typical linear filtering algorithm. It gives a template to the target pixel on the image. The template contains its surrounding adjacent pixels (there are 8 pixels around the target pixel to form a filtering template, which remove the target pixel itself). And then the original pixel value is replaced by the average of all the pixels in the template. Mean filtering is to solve the problem of small point noise in a part of the region, namely, a small matrix range with pixel as the unit. However, the case of flocculent material impurities in transparent soil belongs to the category of larger macroscopic region, so mean filtering will not have any impact on it.

Note that the role of manual exposure combined with reference dots is extremely important for the consistency of color/lighting of all the images. We adjusted the RGB value of the entire image matrix to the standard value according to the RGB value of the feature dots (that is, the reference dots) by using image processing software (such as Photoshop). This procedure will guarantee the consistency of color/lighting of all the images. Since the color of the reference dots do not change, any observed changes in pixel intensity of the black or white portions of the reference dots can be related to a change in lighting either from the light source or from temporary shadows cast on the column. The pixel intensity of the reference dots for each picture taken can therefore be used to correct for changes in pixel intensity of soil that were not a direct result of saturation changes [23].

4.2.2. Background Subtraction. The next operation is to subtract the average pixel intensity value obtained in the previous step from the background, which is a systematic coordination processing between the pictures based on the black and white reference dots. For each image, the procedure subtracts the background intensity value pixel by pixel to obtain the corrected intensity. That is

$$
I(x, y, t)=I_{0}(x, y, t)-I_{0}\left(x_{0}, y_{0}, t\right),
$$

where $I(x, y, t)$ is the corrected pixel intensity; $I_{0}(x, y, t)$ is the initial average value of the pixel intensity obtained after average operation; and $I_{0}\left(x_{0}, y_{0}, t\right)$ is the pixel intensity of the reference dot influenced by the same environmental factors such as the light source.

Note that the same operation should also be performed on the $I_{\mathrm{s}}(x, y, t)$ (pixel intensity at full saturation) and $I_{\mathrm{D}}(x$, $y, t$ ) (pixel intensity at full drying) involved in the next step (normalization).

4.2.3. Normalization. Then, the image pixel intensity was normalized according to the following formula to get the normalized pixel intensity $I_{\mathrm{N}}(x, y, t)$ : 


$$
I_{\mathrm{N}}(x, y, t)=\frac{I_{\mathrm{D}}(x, y, t)-I(x, y, t)}{I_{\mathrm{D}}(x, y, t)-I_{\mathrm{s}}(x, y, t)},
$$

where $I_{\mathrm{N}}(x, y, t)$ is the normalized pixel intensity of the column, $I(x, y, t)$ is the pixel intensity of the image (based on the gray value of the captured image), $I_{\mathrm{s}}(x, y, t)$ is the pixel intensity of the soil column at $100 \%$ saturation, and $I_{\mathrm{D}}$ $(x, y, t)$ is the pixel intensity at fully dry conditions. $I(x, y, t)$, $I_{\mathrm{S}}(x, y, t)$, and $I_{\mathrm{D}}(x, y, t)$ varied between 0 (black) and 255 (white), while $I_{\mathrm{N}}(x, z, t)$ varied between 0 and 1 . All pixel intensities are local values within the range of a single pixel (1.8 pixels $/ \mathrm{mm}$ ). In this study, all the pixel intensities have been taken as average values, for the convenience of fitting calibration with soil saturation. Camera settings were carefully selected to ensure that the images of black and white reference dots are not pixel intensities of 0 or 255, respectively, (not pure black or white) to ensure that the intensity values measured near these extremes are not truncated [23].

Normalized pixel intensity $I_{\mathrm{N}}$ is a crucial tool to quantify the intensity, between saturated and dry extreme conditions. It was first defined by Selker et al. [38]. The similar equation (5) has been used in other studies in transparent porous media and light transmission $[5,23,25,37,39]$. In this study, it is important to note that the pixel intensity is darkest when it is utterly saturated due to the black background behind the column, while the opposite is true for light transmission. This step is critical to correct for variations in brightness due to possible light fluctuations during the experiment.

In Figures 4 and 1(a), you will see some white speckles, which are mainly air bubbles and a little bit of white flocculent impurities in fused quartz, even in almost saturated state. As a matter of fact, it is impossible to remove all the impurities in transparent soils, although active operations such as rinse can be carried out prior to the preparation of transparent soils. Fortunately, normalization can alleviate this problem to a large extent. After that procedure, the influence of interference factors can be effectively excluded, whereas the system variable of optical transmittance can still be effectively measured. Thus, the influence on the purity of experiment materials can be greatly reduced, which highlights the applicability of normalization.

\section{Results and Discussion}

The calibration relationship between the saturation $S_{\mathrm{w}}$ and normalized intensity $I_{\mathrm{N}}$ can be obtained using the above procedure, and the results are shown in Figure 5. In the figure, the black squares represent our experimental data, while the white circles represent the data obtained in previous studies of Peters et al. [23] and Sills et al. [25]. The red solid line represents the fitting curve of all the data in Figure 5. The relationship between saturation and normalized pixel intensity exhibits an exponential function, and they fit well, as described in the following equation:

$$
\begin{aligned}
S_{\mathrm{W}} & =1.01501-1.08248 \cdot e^{-3.438 I_{\mathrm{N}},} \\
R^{2} & =0.98 .
\end{aligned}
$$

For the nonlinearity of the relationship between saturation and normalized pixels $\left(S_{\mathrm{w}}-I_{\mathrm{N}}\right)$ in transparent soils, researchers have reported similar results $[5,23,25]$. Peters et al. [23] presented it as a sequence of piecewise linear functions, as indicated by the green dotted line in Figure 5. The results were based on hanging column drainage experiments using $1.2 \mathrm{~cm}$-high samples in which changes in wetting fluid saturation were calculated from measurements of displaced fluid volume. Pixel intensity was based on the average intensity of an image of the drained layer. Sills et al [25] developed another calibration procedure and found that there was a log-linear relationship between wetting fluid saturation and normalized pixel intensity, as shown by dotted blue line in Figure 5 .

However, in this study, we only aim to find the rules of two-dimensional moisture profile of one-dimensional seepage column, without considering the nonlinear characteristics of its three-dimensional effect. The intensity "noise" probably was caused by the third dimension effect, and there may be some correlation of that effect with the nonlinear law of Figure 5.

Sills et al. [25] proposed a general formula, i.e., equation (7), which can be applied to a variety of conditions, including different fluid pairs, transparent media, model constructions, and lighting conditions:

$$
S_{\mathrm{w}}=\left[\frac{S_{\mathrm{r}}-1}{\ln \left(I_{\mathrm{Nr}}\right)}\right] \ln \left(I_{N}\right)+1,
$$

where $S_{\mathrm{r}}$ is the residual wetting fluid saturation at the end of drainage and $\mathrm{I}_{\mathrm{Nr}}$ is the average pixel intensity of an image (across its width and height) captured under residual wetting fluid conditions.

Therefore, we selected one of the residual states at random, for example, $S_{\mathrm{r}}=0.086$ and the corresponding $I_{\mathrm{Nr}}=0.051$, and substitute that into equation (7). Then, we obtained the corresponding calculated curve (the dotted red line in Figure 6). As seen from Figure 6, the experimental data in this study are fairly consistent with the existing prediction equation (7).

Furthermore, as is seen in Figure 5, the correlation between $S_{\mathrm{w}}$ and $I_{\mathrm{N}}$ in our study shows better agreement than previous research work [23, 25], whose correlation coefficient's square is 0.98 . The traditional overflow method could not overcome the limitations of nonuniformity in the seepage process, which may be the essential reason for low correlation in these two previous studies. In addition, we also find that the fitting curve of our study has a certain upward shift compared with the curve predicted by Sills et al. [25], which may have resulted from the different particle grades of transparent sand selected in the two tests (as shown in Table 1).

In fact, the image intensity of saturated sand can be changed by both depth and particles size. And there is an exiting condition that the image intensities are different although the saturation is same. To solve this, two aspects should be taken into account: firstly, the visuality of transparent soils is closely related to the depth. In general, the 


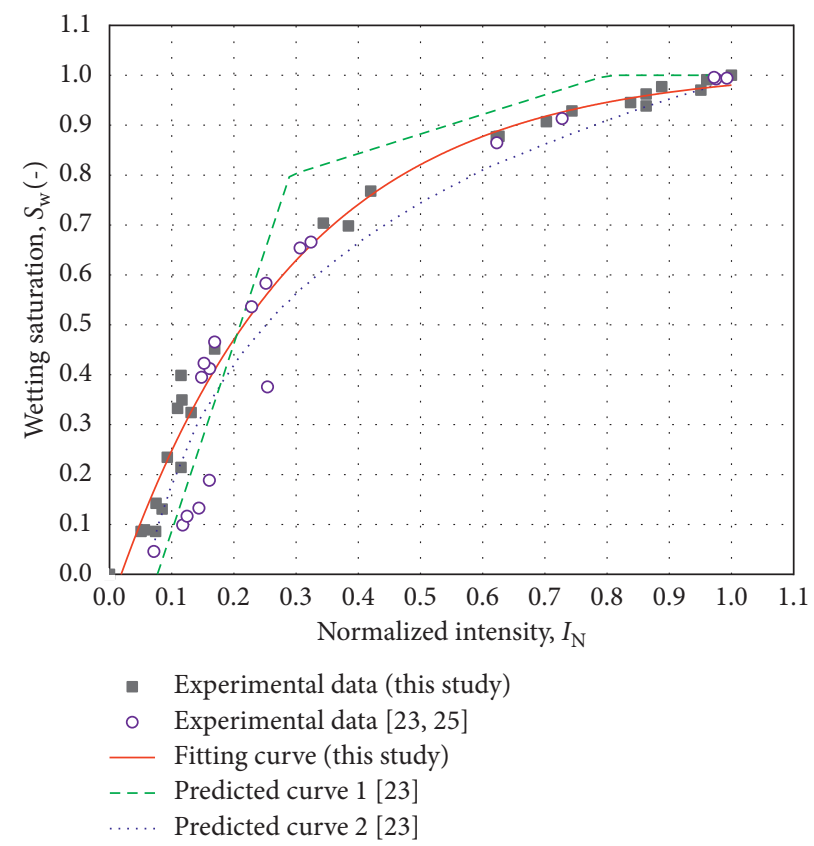

Figure 5: Relationship between saturation $\left(S_{\mathrm{w}}\right)$-normalized pixel intensity $\left(I_{\mathrm{N}}\right)$, compared with measured saturation-intensity pairs in the study of Peters et al. [23] and Sills et al. [25].

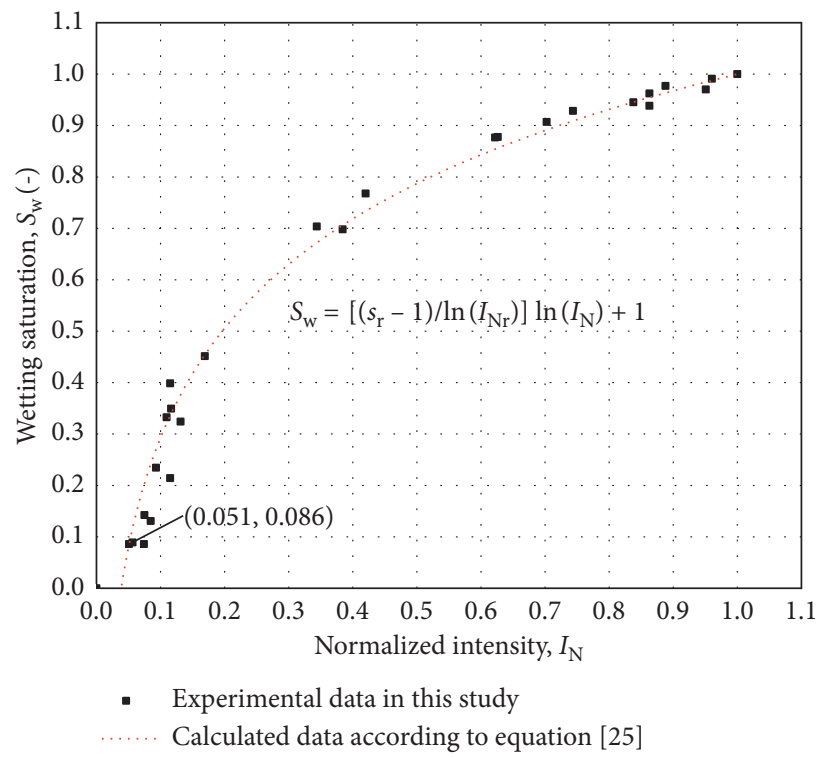

Figure 6: Correlation between experimental data and the general formula (cited by [25]).

thinner the depth, the better the visuality. In our research, the depth of column was designed within $50 \mathrm{~mm}$, and the effects of depth on the image intensities can be negligible for the saturated sand. In brief, we aimed to observe the $2 \mathrm{D}$ flow effect without considering the saturation change throughout the depth; secondly, there is only one-grain gradation of transparent sand used to eliminate the impact of particles size in this study. So, the $S_{\mathrm{w}}-I_{\mathrm{N}}$ relationship in this study may be a little different from that of other grain gradation, as seen in Figure 5. Therefore, as for the applicable condition of this research, the transparent sand with one given gradation is used in our research with the thickness of $50 \mathrm{~mm}$. As for the transparent soils with different gradations, the image intensities could be a little different, which needs to be further explored in future.

\section{Numerical Example}

According to the above image analysis method, the water content map of a certain drainage process time $(t=0: 0: 0,0$ : $33: 40,0: 38: 25,0: 42: 20,0: 51: 20,1: 26: 10$, and 2:53:50) (see Figure 7) was predicted using MATLAB software. The 


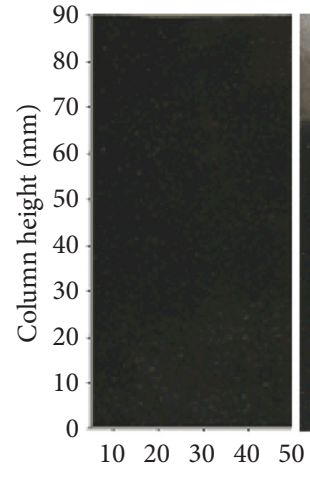

(A) $t=0: 0: 0$

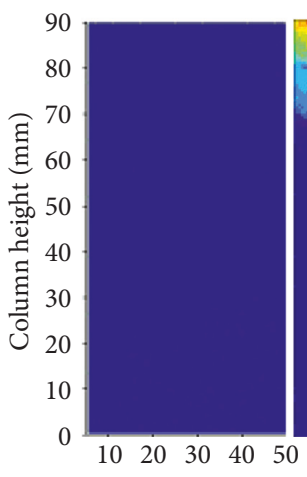

(A) $t=0: 0: 0$

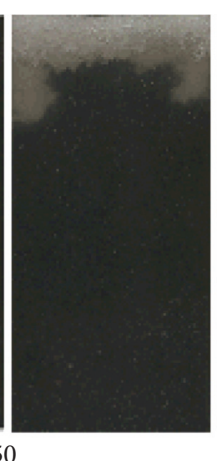

(B) $t=0: 33: 40$

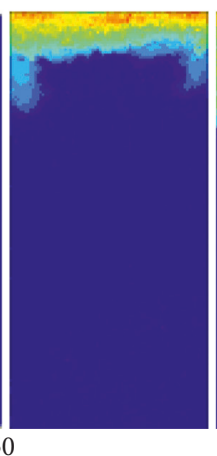

(B) $t=0: 33: 40$

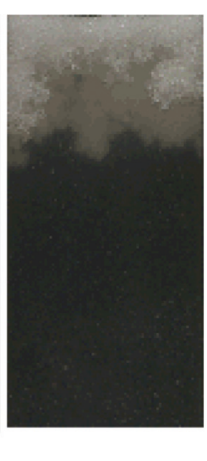

(C) $t=0: 38: 25$

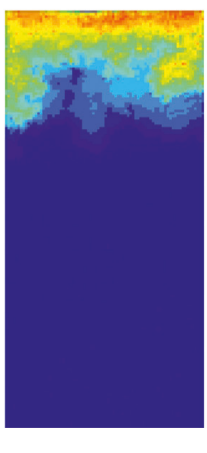

(C) $t=0: 38: 25$

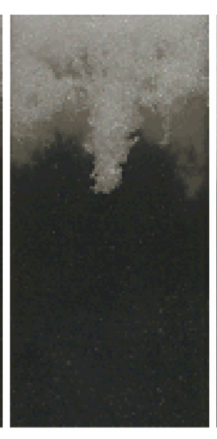

(D) $t=0: 42: 20$

(a)

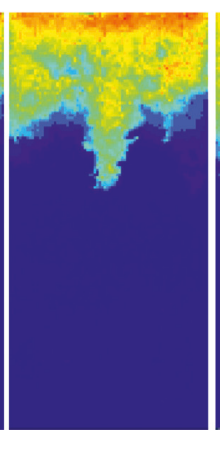

(D) $t=0: 42: 20$

(b)

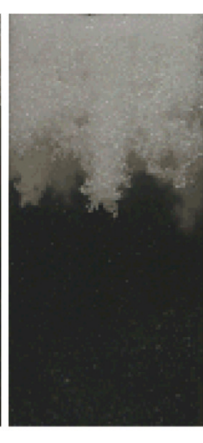

(E) $t=0: 51: 20$

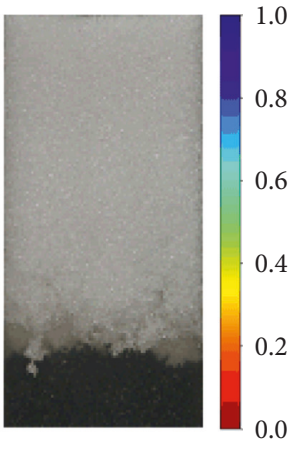

(G) $t=2: 53: 50$

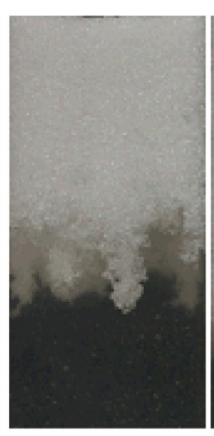

(F) $t=1: 26: 10$

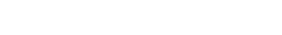

FiguRE 7: Visualization of the fingering phenomena at the air front through partially saturated transparent soil at different drainage stages: (a) original digital photographs and (b) moisture maps from photometric procedure.

original digital photos of soil column are shown in Figure $7(\mathrm{a})$, and the moisture map after the photometric treatment is given in Figure $7(\mathrm{~b})$. From the photos, the nonuniformity of the water profile and the irregularity of the boundary during the seepage process are clearly seen. The actual size of the object model is $90 \mathrm{~mm} \times 50 \mathrm{~mm}$ (height $\times$ width), and the division precision is 20 pixels. Figure 7 indicates that the moisture profile obtained from (4) fits well with the digital photos.

In view of the heterogeneity of the water profile and the irregularity of the boundary in the actual seepage process, traditional seepage theory research is mostly confined to the layered soil, which requires the installation of at least one moisture probe in each representative soil layer to achieve significant changes in water measurement $[37,40]$ as well as to construct calibration curves associated with each type of soil. In contrast, this photometric method has a stronger adaptability to the nonuniform transient effect of the above unsaturated seepage process, which provides a new perspective for the verification of the numerical model in the laboratory seepage test.

Another remarkable characteristic of this photometric procedure is that it is intended to be efficiently applied to a large number of digital images with high temporal and spatial resolution, which required much less computation. This would make it possible to put benchmarks in place for unsaturated codes. That is, it would no longer be based solely on local measurements of pressure head, water content, or water content maps. Its application may even be expanded to the study of hydrodynamic effects and surface evaporation.

\section{Conclusion}

This paper aims to seek a novel method to quantify liquid saturation in the flow of partially saturated porous media using transparent soil. An improved calibration procedure of the saturation-pixel intensity relationship was proposed, which is crucial to the application of transparent soil and optical imaging technologies in the quantitative analysis of unsaturated soil flow. It overcomes the limitation of the irregular boundary of the wetting liquid in a residual state caused by the gravity-driven effects. Based on this, a photometric procedure was developed to efficiently quantifying the water maps during drainage stages, which were applied to a large number of digital images with high temporal and spatial resolution and required much less computation. We hope our study results will expand further application of transparent soil in quantifying the fluid saturation in the partially saturated flow.

\section{Data Availability}

The figures and tables data used to support the findings of this study are included within the article. 


\section{Conflicts of Interest}

The authors declare that there are no conflicts of interest regarding the publication of this paper.

\section{Acknowledgments}

This study was partially supported by the Natural Key Basic Research Development Plan of China (973 Program No. 2014CB744701) and the Fundamental Research Funds for the Central Universities (No. lzujbky-2017-170). The authors would like to express their heartfelt gratitude to all the members who helped and cooperated with us.

\section{References}

[1] H. Vereecken, J. A. Huisman, H. Bogena, J. Vanderborght, J. A. Vrugt, and J. W. Hopmans, "On the value of soil moisture measurements in vadose zone hydrology, a review," Water Resources Research, vol. 44, no. 4, pp. 253-270, 2008.

[2] H. Vereecken, A. Schnepf, J. W. Hopmans et al., "Modeling soil processes: review, key challenges and new perspectives," Vadose Zone Journal, vol. 15, no. 5, pp. 1-57, 2016.

[3] S. L. Susha Lekshmi, D. N. Singh, and M. Shojaei Baghini, "A critical review of soil moisture measurement," Measurement, vol. 54, no. 8, pp. 92-105, 2014.

[4] N. T. Hoa, "A new method allowing the measurement of rapid variations of the water content in sandy porous media," Water Resources Research, vol. 17, no. 1, pp. 41-48, 1981.

[5] V. C. Tidwell and R. J. Glass, " $\mathrm{X}$ ray and visible light transmission for laboratory measurement of two-dimensional saturation fields in thin-slab systems," Water Resources Research, vol. 30, no. 11, pp. 2873-2882, 1994.

[6] M. R. Niemet and J. S. Selker, "A new method for quantification of liquid saturation in 2D translucent porous media systems using light transmission," Advances in Water Resources, vol. 24, no. 6, pp. 651-666, 2001.

[7] M. Oostrom, J. H. Dane, and T. W. Wietsma, "A review of multidimensional, multifluid, intermediate-scale experiments: flow behavior, saturation imaging, and tracer detection and quantification," Vadose Zone Journal, vol. 6, no. 3, pp. 610-637, 2007.

[8] C. J. Werth, C. Zhang, M. L. Brusseau, and M. T. Oostrom, “A review of non-invasive imaging methods and applications in contaminant hydrogeology research," Journal of Contaminant Hydrology, vol. 113, no. 1-4, pp. 1-24, 2010.

[9] R. J. Mannheimer and C. J. Oswald, "Development of transparent porous media with permeabilities of soils and reservoir materials," Ground Water, vol. 31, no. 5, pp. 781-788, 1993.

[10] M. Iskander, J. Lai, C. Oswald, and R. Mannheimer, "Development of a transparent material to model the geotechnical properties of soil," Geotechal Testing Journal, vol. 17, no. 4, pp. 425-433, 1994.

[11] M. Iskander and J. Liu, "Spatial deformation measurement using transparent soil," Geotechal Testing Journal, vol. 33, no. 4, pp. 1-7, 2010.

[12] M. Iskander, R. J. Bathurst, and M. Omidvar, "Past, present, and future of transparent soils," Geotechnical Testing Journal, vol. 38, no. 5, pp. 393-401, 2015.

[13] A. Welker, J. Bowders, and R. Gilbert, “Applied research using a transparent material with hydraulic properties similar to soil," Geotechniacl Testing Journal, vol. 22, no. 3, pp. 266-270, 1999.

[14] J. Liu, M. Iskander, K. Tabe, and K. Kosterelos, "Flow visualization using transparent sythetic soils," in Proceedings of the International Conference on Soil Mechanics and Geotechnial Engineering, pp. 2411-2414, Osaka, Japan, September 2006.

[15] L. D. Suits, T. C. Sheahan, H.-C. Lo et al., "A transparent water-based polymer for simulating multiphase flow," Geotechnical Testing Journal, vol. 33, no. 1, pp. 1-13, 2009.

[16] W. Sui, H. Qu, and Y. Gao, "Modeling of grout propagation in transparent replica of rock fractures," Geotechnical Testing Journal, vol. 38, pp. 765-773, 2015.

[17] Y. Gao, W. Sui, and J. Liu, "Visualization of chemical grout permeation in transparent soil," Geotechnical Testing Journal, vol. 38, no. 5, pp. 774-786, 2015.

[18] K. Tabe, "Transparent aquabeads to model LNAPL ganglia migration through surfactant flushing," Geotechnical Testing Journal, vol. 38, no. 5, pp. 787-804, 2015.

[19] M. A. Theodoropoulou, V. Karoutsos, C. Kaspiris, and C. D. Tsakiroglou, "A new visualization technique for the study of solute dispersion in model porous media," Journal of Hydrology, vol. 274, no. 1-4, pp. 176-197, 2003.

[20] H. Ovdat and B. Berkowitz, "Pore-scale study of drainage displacement under combined capillary and gravity effects in index-matched porous media," Water Resources Research, vol. 42, no. 6, pp. 411-425, 2006.

[21] X.-Z. Kong, M. Holzner, F. Stauffer, and W. Kinzelbach, "Time-resolved 3D visualization of air injection in a liquidsaturated refractive-index-matched porous medium," Experiments in Fluids, vol. 50, no. 6, pp. 1659-1670, 2011.

[22] S. Kashuk, R. Mercurio, and M. Iskander, "Methodology for optical imaging of NAPL 3D distribution in transparent porous media," Geotechnical Testing Journal, vol. 38, no. 5, pp. 603-619, 2015.

[23] S. B. Peters, G. A. Siemens, and W. A. Take, "Characterization of transparent soil for unsaturated applications," Geotechnical Testing Journal, vol. 34, no. 5, pp. 445-456, 2011.

[24] G. A. Siemens, S. B. Peters, and W. A. Take, "Comparison of confined and unconfined infiltration in transparent porous media," Water Resources Research, vol. 49, no. 2, pp. 851-863, 2013.

[25] L. K. Sills, K. G. Mumford, and G. A. Siemens, "Quantification of fluid saturations in transparent porous media," Vadose Zone Journal, vol. 16, no. 2, pp. 1-9, 2017.

[26] G. A. Siemens and R. A. Beddoe, "Spatial resolution of degree of saturation measurements in unsaturated transparent soil experiments," in Proceedings of 2nd Pan-merican Conference on Unsaturated Soils, Geotechnical Special Publication (PanAm Unsaturated Soils 2017 GSP), vol. 300, pp. 174-184, Dallas, TX, USA, 2018.

[27] R. Lagioia, A. Sanzeni, and F. Colleselli, "Air, water and vacuum pluviation of sand specimens for the triaxial apparatus," Soils and Foundations, vol. 46, no. 1, pp. 61-67, 2006.

[28] R. J. Glass, T. S. Steenhuis, and J.-Y. Parlange, "Mechanism for finger persistence in homogeneous, unsaturated, porous media," Soil Science, vol. 148, no. 1, pp. 60-70, 1989.

[29] J. Selker, J.-Y. Parlange, and T. Steenhuis, "Fingered flow in two dimensions: 2. Predicting finger moisture profile," Water Resources Research, vol. 28, no. 9, pp. 2523-2528, 1992.

[30] D. A. DiCarlo, "Experimental measurements of saturation overshoot on infiltration," Water Resources Research, vol. 40, no. 4, pp. 1-9, 2004.

[31] F. M. Ezzein and R. J. Bathurst, “A new approach to evaluate soil-geosynthetic interaction using a novel pullout test 
apparatus and transparent granular soil," Geotextiles and Geomembranes, vol. 42, no. 3, pp. 246-255, 2014.

[32] M. Omidvar, J. D. Malioche, Z. Chen, M. Iskander, and S. Bless, "Visualizing kinematics of dynamic penetration in granular media using transparent soils," Geotechniacl Testing Journal, vol. 38, no. 5, pp. 1-18, 2015.

[33] F. M. Ezzein and R. J. Bathurst, "A transparent sand for geotechnical laboratory modeling," Geotechal Testing Journal, vol. 34, no. 6, pp. 1-12, 2011.

[34] D. J. White, W. A. Take, and M. D. Bolton, "Soil deformation measurement using particle image velocimetry (PIV) and photogrammetry," Géotechnique, vol. 53, no. 7, pp. 619-631, 2003.

[35] C. Chini, J. Wallace, C. Rutherford, and C. J. Peschel, "Shearing failure visualization using digital image correlation and particle image velocimetry in soft clay using a transparent soil," Geotechal Testing Journal, vol. 38, no. 5, pp. 1-17, 2015.

[36] W. A. Take, "Thirty-sixth Canadian geotechnical colloquium: advances in visualization of geotechnical processes through digital image correlation," Canadian Geotechnical Journal, vol. 52, no. 9, pp. 1199-1220, 2015.

[37] B. Belfort, S. Weill, and F. Lehmann, "Image analysis method for the measurement of water saturation in a two-dimensional experimental flow tank," Journal of Hydrology, vol. 550, pp. 343-354, 2017.

[38] J. S. Selker, T. S. Steenhuis, and J. Y. Parlange, Preferential Flow in Homogeneous Sandy Soils without Layering, American Society of Agricultural Engineers, St. Joseph, MI, USA, 1989.

[39] M. Konz, P. Ackerer, E. Meier, P. Huggenberger, E. Zechner, and D. Gechter, "On the measurement of solute concentrations in 2-D flow tank experiments," Hydrology and Earth System Sciences, vol. 12, no. 3, pp. 727-738, 2008.

[40] S. S. Zanetti, R. A. Cecílio, E. G. Alves, V. H. Silva, and E. F. Sousa, "Estimation of the moisture content of tropical soils using colour images and artificial neural networks," Catena, vol. 135, pp. 100-106, 2015.

[41] B. R. Munson, D. F. Yound, and T. H. Okiishi, Fundamentals of Fluid Mechanics, John Wiley \& Sons, New York, NY, USA, 1998. 


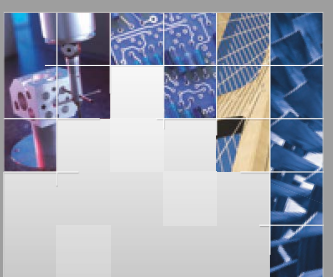

\section{Enfincering}
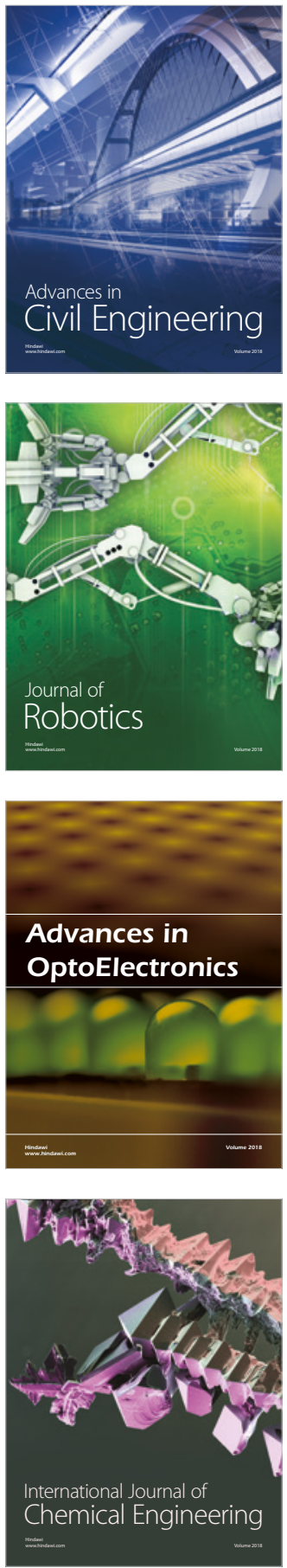

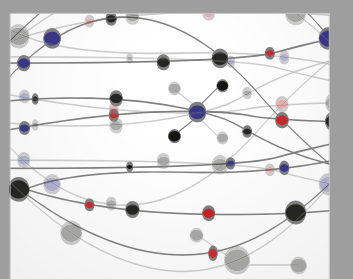

\section{Rotating \\ Machinery}

The Scientific World Journal

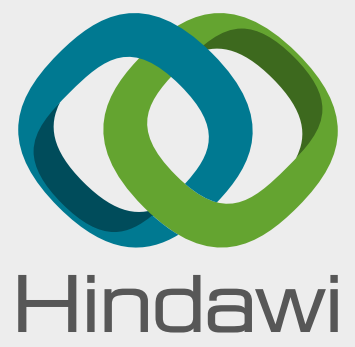

Submit your manuscripts at

www.hindawi.com
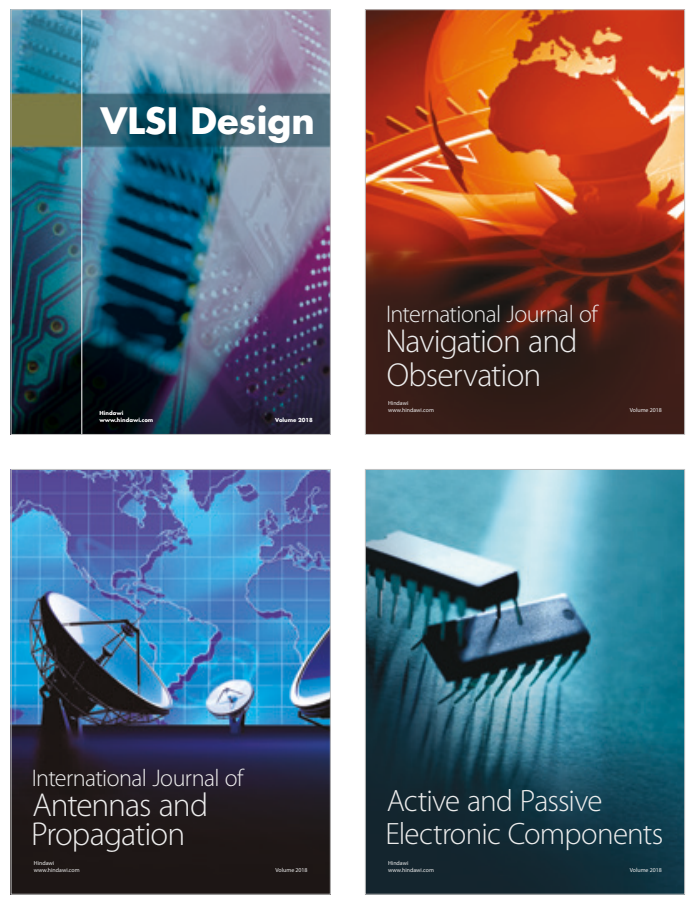
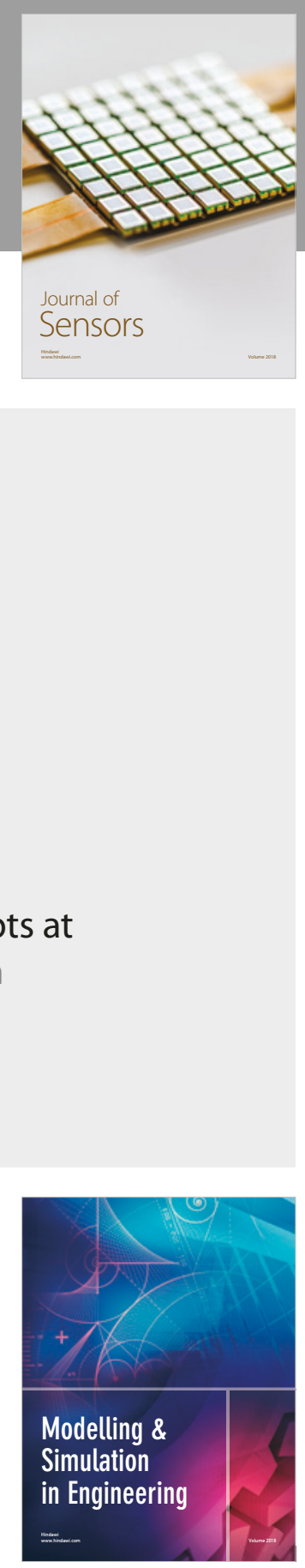

\section{Advances \\ Multimedia}
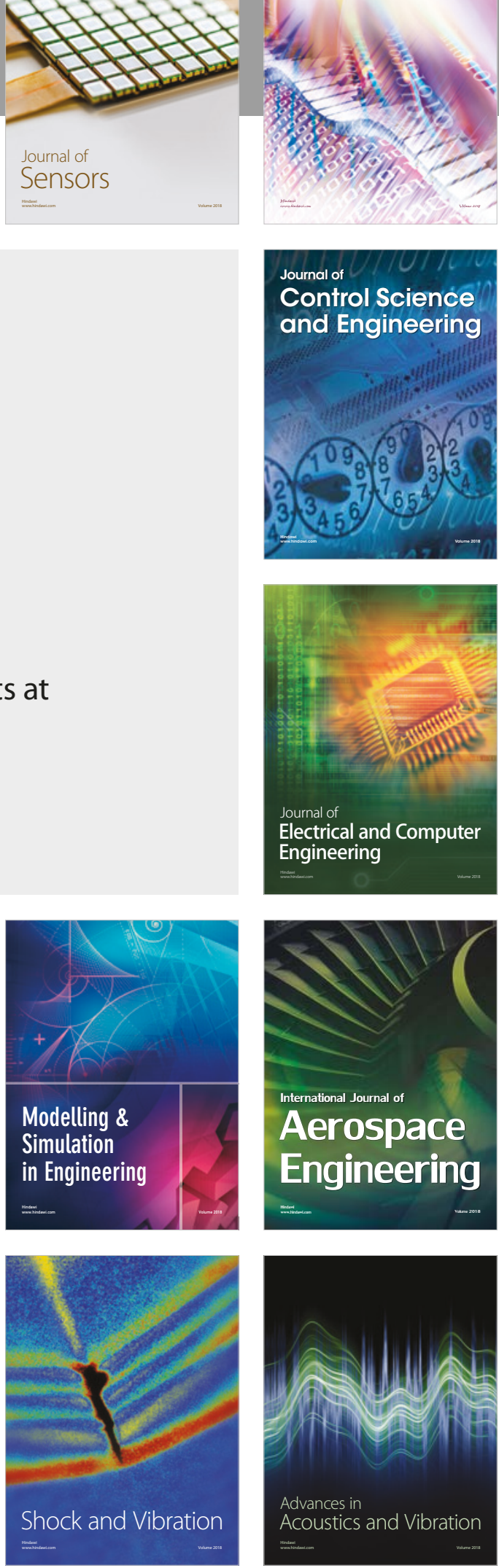